\title{
Roles of Hund's rule coupling in excitonic density-wave states
}

\author{
Tatsuya Kaneko and Yukinori Ohta \\ Department of Physics, Chiba University, Chiba 263-8522, Japan
}

(Dated: Received 17 July 2014)

\begin{abstract}
Excitonic density-wave states realized by the quantum condensation of electron-hole pairs (or excitons) are studied in the two-band Hubbard model with Hund's rule coupling and the pair hopping term. Using the variational cluster approximation, we calculate the grand potential of the system and demonstrate that Hund's rule coupling always stabilizes the excitonic spin-densitywave state and destabilizes the excitonic charge-density-wave state and that the pair hopping term enhances these effects. The characteristics of these excitonic density-wave states are discussed using the calculated single-particle spectral function, density of states, condensation amplitude, and pair coherence length. Implications of our results in the materials' aspects are also discussed.
\end{abstract}

PACS numbers: 71.10.Fd, 71.35.-y, 75.30.Fv, 71.30.+h

\section{INTRODUCTION}

The excitonic phases, often referred to as the excitonic insulator states or excitonic density-wave states, are described by the quantum condensation of excitons, which were predicted to occur in a small band-gap semiconductor or a small band-overlap semimetal $\underline{\underline{1}-3}$ The exciton condensation in semimetallic systems can be described in analogy with the BCS theory of superconductors, and that in semiconducting systems can be discussed in terms of the Bose-Einstein condensation (BEC) of preformed excitons. ${ }^{4}$ The crossover phenomena between the BCS and the BEC states are then expected to produce rich physics in the field of quantum many-body systems. The excitonic phases are characterized by an order parameter $\left\langle c_{\boldsymbol{k}+\boldsymbol{Q} \sigma}^{\dagger} f_{\boldsymbol{k} \sigma^{\prime}}\right\rangle$, where $c_{\boldsymbol{k} \sigma}^{\dagger}$ and $f_{\boldsymbol{k} \sigma}^{\dagger}$ are the creation operators of an electron in the conduction and valence bands, respectively. If the valence-band top and conductionband bottom are separated by the wave vector $\boldsymbol{Q}$, the system shows the density wave with modulation $\boldsymbol{Q}^{2,3}$

A number of candidate materials for the excitonic phases have been discovered. It was claimed that $\mathrm{Tm}(\mathrm{Se}, \mathrm{Te})$ shows a transition into the excitonic insulator state by applying pressure ${ }^{\frac{5}{5}}$ The weak ferromagnetism of $\mathrm{Ca}_{1-x} \mathrm{La}_{x} \mathrm{~B}_{6}$ was interpreted in terms of a doped spin-triplet excitonic insulator ${ }^{6-8}$ Recently, the phase transition of a layered chalcogenide $\mathrm{Ta}_{2} \mathrm{NiSe}_{5}$ has been attributed to a realization of the spin-singlet excitonic insulator and has attracted much experimental and theoretical attention. ${ }^{\frac{9}{-12}}$ The charge-density wave (CDW) of $1 T-\mathrm{TiSe}_{2}$ has also been claimed to be of the excitonic origin. ${ }^{13-16}$ The spin-density wave (SDW) state of iron pnictide superconductors has sometimes been argued to be of the excitonic origin as well. ${ }^{17-19}$ It was proposed that the condensation of spin-triplet excitons can occur in the proximity of a spin-state transition; ${ }^{20}$ $\mathrm{Pr}_{0.5} \mathrm{Ca}_{0.5} \mathrm{CoO}_{3}$ is an example. ${ }^{21}$

In this paper, motivated by the above development in the field, we study the stability of the excitonic density-wave states in the two-band Hubbard model where Hund's rule coupling $J$, the pair hopping term $J^{\prime}$, as well as the interorbital Coulomb repulsion $U^{\prime}$ are taken into account in addition to the standard intraorbital Hubbard repulsion $U$. It is known that the interorbital repulsion $U^{\prime}$ induces the excitonic instability in the system, 19,22 but the condensations of the spin-singlet and spin-triplet excitons are exactly degenerate unless Hund's rule coupling, the pair hopping term, or electron-phonon coupling are taken into account. Thus, we here study the roles of Hund's rule coupling and the pair hopping term played in the excitonic density wave of which not much is known so far.

We first rewrite the interorbital interaction terms of the Hamiltonian in terms of the creation and annihilation operators of the spin-singlet and spin-triplet excitons. We then show that the interorbital repulsion $U^{\prime}$ actually leads to the exciton formation in both the spinsinglet and the spin-triplet channels and that Hund's rule coupling always lowers the energy of the spin-triplet exciton and raises the energy of the spin-singlet exciton. The variational cluster approximation (VCA) ${ }^{23,24}$ is then used to study the two-band Hubbard model in detail, and we show that Hund's rule coupling and the pair hopping term always stabilize the excitonic SDW state and destabilize the excitonic CDW state. The characteristics of these excitonic density-wave states will moreover be examined using a variety of calculated physical quantities, including the single-particle spectral function, density of states (DOS), condensation amplitude, and pair coherence length. Consequences of the present results on the excitonic density-wave states of a variety of materials will also be discussed.

This paper is organized as follows: In Sec. II, the model and method of calculations will be given. In Sec. III, the results of calculations for various physical quantities will be presented. Summary and discussion will be given in Sec. IV.

\section{MODEL AND METHOD}

\section{A. The two-band Hubbard model}

We consider the two-band Hubbard model defined by the Hamiltonian,

$$
\begin{aligned}
\mathcal{H}= & -t \sum_{\langle i, j\rangle} \sum_{\sigma} \sum_{\alpha} \alpha_{i \sigma}^{\dagger} \alpha_{j \sigma}-D \sum_{i}\left(n_{i f}-n_{i c}\right) \\
& +U \sum_{i} \sum_{\alpha} n_{i \alpha \uparrow} n_{i \alpha \downarrow}+U^{\prime} \sum_{i} n_{i f} n_{i c} \\
& -2 J \sum_{i}\left(\boldsymbol{S}_{i f} \cdot \boldsymbol{S}_{i c}+\frac{1}{4} n_{i f} n_{i c}\right) \\
& -J^{\prime} \sum_{i}\left(f_{i \uparrow}^{\dagger} f_{i \downarrow}^{\dagger} c_{i \uparrow} c_{i \downarrow}+c_{i \uparrow}^{\dagger} c_{i \downarrow}^{\dagger} f_{i \uparrow} f_{i \downarrow}\right)
\end{aligned}
$$


where $\alpha_{i \sigma}^{\dagger}\left(=f_{i \sigma}^{\dagger}, c_{i \sigma}^{\dagger}\right)$ denotes the creation operator of an electron with spin $\sigma(=\uparrow, \downarrow)$ on the $\alpha(=f, c)$ orbital at site $i$ and $n_{i \alpha}=n_{i \alpha \uparrow}+n_{i \alpha \downarrow}=\alpha_{i \uparrow}^{\dagger} \alpha_{i \uparrow}+\alpha_{i \downarrow}^{\dagger} \alpha_{i \downarrow} \cdot t$ is the hopping integral between the same orbitals on the neighboring sites, and $D$ is the level splitting between the two orbitals. $U$ and $U^{\prime}$ are the intra- and interorbital Coulomb repulsions, respectively, between electrons and $J$ and $J^{\prime}$ are the strengths of Hund's rule coupling and the pair hopping term, respectively. Note that $J=J^{\prime}$ in the standard two-orbital Hubbard model, but when necessary we retain only Hund's rule coupling by setting $J^{\prime}=0$ to examine the role of the pair hopping term. Throughout the paper, we assume the filling of two electrons per site (or half-filling), i.e., $\left\langle n_{f}\right\rangle+\left\langle n_{c}\right\rangle=2$, where $\left\langle n_{\alpha}\right\rangle=\sum_{i, \sigma}\left\langle n_{i \alpha \sigma}\right\rangle / N$.

The Hamiltonian Eq. (1) in the spinless case with $U=J=J^{\prime}=0$ is equivalent to the extended FalicovKimball model with dispersive $c$ and $f$ electrons of which the excitonic insulator state has been studied much in detail. $25-29$ The excitonic phases in the two-band Hubbard model without Hund's rule coupling and the pair hopping term $\left(J=J^{\prime}=0\right)$ have also been studied recently 19,22 where it was shown that the model exhibits three ground-state phases: (i) the band insulator (at $\left.U^{\prime}, D \gg U, J\right)$, where $\left\langle n_{f}\right\rangle=2$ and $\left\langle n_{c}\right\rangle=0$, (ii) the antiferromagnetic Mott insulator (at $U, J \gg U^{\prime} D$ ), where $\left\langle n_{f}\right\rangle=\left\langle n_{c}\right\rangle=1$, and (iii) the excitonic density-wave state between the above two phases, where $2>\left\langle n_{f}\right\rangle>$ $1>\left\langle n_{c}\right\rangle>0$. However, although many studies have been performed on the multiband Hubbard models, recently in relation to iron pnictide superconductors, $30-33$ the excitonic density-wave states in the two-band Hubbard model with Hund's rule coupling have not been studied in detail; only a recent dynamical mean-field theory (DMFT) calculation ${ }^{20,21}$ is noticed.

To see the stability of the spin-singlet and spin-triplet excitons, let us introduce the creation operators of the spin-singlet and spin-triplet excitons, respectively, which are defined as

$$
A_{i}^{0^{\dagger}}=\frac{1}{\sqrt{2}} \sum_{\sigma} c_{i \sigma}^{\dagger} f_{i \sigma}, \quad \boldsymbol{A}_{i}^{\dagger}=\frac{1}{\sqrt{2}} \sum_{\sigma \sigma^{\prime}} c_{i \sigma}^{\dagger} \boldsymbol{\sigma}_{\sigma \sigma^{\prime}} f_{i \sigma^{\prime}},
$$

where $\boldsymbol{\sigma}$ is the vector of the Pauli matrices. Using the spin-singlet and spin-triplet exciton operators thus defined, the interorbital Coulomb repulsion term can be divided exactly into the spin-singlet and spin-triplet terms as

$$
U^{\prime} n_{i f} n_{i c}=-U^{\prime} A_{i}^{0 \dagger} A_{i}^{0}-U^{\prime} \boldsymbol{A}_{i}^{\dagger} \cdot \boldsymbol{A}_{i} .
$$

Therefore, the formation of excitons lowers the energy of the system in both the spin-singlet and the spin-triplet channels by the same amount. Hund's rule coupling and the pair hopping terms can also be rewritten exactly as

$$
\begin{aligned}
-J \boldsymbol{S}_{i f} \cdot \boldsymbol{S}_{i c} & =\frac{3 J}{4} A_{i}^{0^{\dagger}} A_{i}^{0}-\frac{J}{4} \boldsymbol{A}_{i}^{\dagger} \cdot \boldsymbol{A}_{i}, \\
-J^{\prime} c_{i \uparrow}^{\dagger} c_{i \downarrow}^{\dagger} f_{i \uparrow} f_{i \downarrow} & =\frac{J^{\prime}}{4} A_{i}^{0^{\dagger}} A_{i}^{0^{\dagger}}-\frac{J^{\prime}}{4} \boldsymbol{A}_{i}^{\dagger} \cdot \boldsymbol{A}_{i}^{\dagger} .
\end{aligned}
$$

Therefore, due to the Hund's rule coupling term, the formation of the spin-triplet (spin-singlet) excitons always lowers (raises) the energy of the system, thus lifting the degeneracy that occurs at $J=J^{\prime}=0$. The pair hopping term can also be divided into the spin-singlet and spintriplet terms as in Eq. (5), which are of the off-diagonal form.

\section{B. Variational cluster approximation}

We use the $\mathrm{VCA}, \stackrel{23,24}{2}$ which is a quantum cluster method based on the self-energy functional theory, 34 and solve the quantum many-body problem defined in Eq. (11). Note that, unlike in DMFT, we can taken into account the effects of short-range spatial electron correlations precisely in this approach. The VCA introduces the disconnected finite-size clusters that are solved exactly to obtain the exact self-energy of the clusters $\hat{\Sigma}^{\prime}$ with which a superlattice is formed as a reference system. The matrices are indicated by a ${ }^{\wedge}$ hereafter. By restricting the trial self-energy to $\hat{\Sigma}^{\prime}$, we obtain an approximate grand potential of the original system,

$$
\Omega=\Omega^{\prime}-\frac{1}{N} \oint_{C} \frac{\mathrm{d} z}{2 \pi i} \sum_{\boldsymbol{K}, \sigma} \ln \operatorname{det}\left[\hat{I}-\hat{V}_{\sigma}(\boldsymbol{K}) \hat{G}_{\sigma}^{\prime}(z)\right],
$$

where $\Omega^{\prime}$ is the grand potential of the reference system, $\hat{I}$ is the unit matrix, $\hat{V}$ is the hopping matrix between adjacent clusters, and $\hat{G}^{\prime}$ is the exact Green's function of the reference system. The $\boldsymbol{K}$ summation is performed in the reduced Brillouin zone of the superlattice, and the contour $C$ of the frequency integral encloses the negative real axis. Details of the VCA can be found in Refs. 35, 36].

To study the symmetry-breaking phases in the VCA, we introduce the Weiss fields as variational parameters. The variational Hamiltonian for the excitonic CDW and SDW states are then defined as

$$
\begin{aligned}
& \mathcal{H}_{\mathrm{CDW}}^{\prime}=\mathcal{H}+\Delta_{0}^{\prime} \sum_{i, \sigma} e^{i \boldsymbol{Q} \cdot \boldsymbol{r}_{i}}\left(c_{i \sigma}^{\dagger} f_{i \sigma}+\text { H.c. }\right), \\
& \mathcal{H}_{\mathrm{SDW}}^{\prime}=\mathcal{H}+\Delta_{z}^{\prime} \sum_{i, \sigma} \sigma e^{i \boldsymbol{Q} \cdot \boldsymbol{r}_{i}}\left(c_{i \sigma}^{\dagger} f_{i \sigma}+\text { H.c. }\right),
\end{aligned}
$$

respectively, where $\Delta_{0}^{\prime}$ is the Weiss field for condensation of the spin-singlet excitons and $\Delta_{z}^{\prime}$ is the $z$ component of the Weiss field for condensation of the spintriplet excitons. The variational parameters $\Delta_{0}^{\prime}$ and $\Delta_{z}^{\prime}$ are optimized on the basis of the variational principle, i.e., $\partial \Omega / \partial \Delta_{0}^{\prime}=0$ for the excitonic CDW state and $\partial \Omega / \partial \Delta_{z}^{\prime}=0$ for the excitonic SDW state. The solutions with $\Delta_{0}^{z} \neq 0$ and $\Delta_{z}^{\prime} \neq 0$ correspond to the excitonic CDW and SDW states, respectively.

We solve the eigenvalue problem $\mathcal{H}^{\prime}\left|\psi_{0}\right\rangle=E_{0}\left|\psi_{0}\right\rangle$ of a finite-size ( $L_{c}$ sites) cluster to obtain the ground state, and we calculate the trial Green's function by the Lanczos exact-diagonalization method. Using the basis $\boldsymbol{\Psi}_{i}^{\dagger}=\left(f_{i \sigma}^{\dagger}, c_{i \sigma}^{\dagger}\right)$, the Green's-function matrix $\hat{G}_{\sigma}^{\prime}$ in Eq. (6) may be written as

$$
\hat{G}_{\sigma}^{\prime}(\omega)=\left(\begin{array}{cc}
\hat{G}_{\sigma}^{\prime f f}(\omega) & \hat{G}_{\sigma}^{\prime f c}(\omega) \\
\hat{G}_{\sigma}^{\prime c f}(\omega) & \hat{G}_{\sigma}^{\prime c c}(\omega)
\end{array}\right)
$$

where $\hat{G}_{\sigma}^{\prime \alpha \beta}$ is an $L_{c} \times L_{c}$ matrix and each matrix element 
is defined as

$$
\begin{aligned}
G_{i j, \sigma}^{\alpha \beta}(\omega) & =\left\langle\psi_{0}\left|\alpha_{i \sigma} \frac{1}{\omega-\mathcal{H}^{\prime}+E_{0}} \beta_{j \sigma}^{\dagger}\right| \psi_{0}\right\rangle \\
& +\left\langle\psi_{0}\left|\beta_{j \sigma}^{\dagger} \frac{1}{\omega+\mathcal{H}^{\prime}-E_{0}} \alpha_{i \sigma}\right| \psi_{0}\right\rangle .
\end{aligned}
$$

The matrix $\hat{V}$ in Eq. (6) is given as

$$
\hat{V}_{\sigma}(\boldsymbol{K})=\left(\begin{array}{cc}
\hat{T}(\boldsymbol{K}) & -\Delta_{\sigma}^{\prime} \hat{I} \\
-\Delta_{\sigma}^{\prime} \hat{I} & \hat{T}(\boldsymbol{K})
\end{array}\right)
$$

where $\hat{T}(\boldsymbol{K})$ is the intercluster hopping matrix with the matrix elements $T_{i j}(\boldsymbol{K})=$ $-t \sum_{\boldsymbol{X}, x} e^{i \boldsymbol{K} \cdot \boldsymbol{X}} \delta_{i+x, j} \delta_{\boldsymbol{R}+\boldsymbol{X}, \boldsymbol{R}^{\prime}}$. Here, $x$ denotes the neighboring site of the $i$ th site, and $\boldsymbol{X}$ denotes the neighboring cluster of the $\boldsymbol{R}$ th cluster. $\Delta_{\sigma}^{\prime}=\Delta_{0}^{\prime}$ for the excitonic CDW state, and $\Delta_{\sigma}^{\prime}=\sigma \Delta_{z}^{\prime}$ for the excitonic SDW state.

In our VCA calculation, we assume the twodimensional square lattice and use an $L_{c}=2 \times 2=4$ site (eight-orbital) cluster as the reference system. We set $D / t=3.2$ so that the noninteracting tight-binding band structure is a semimetal with a small band overlap. The band structure has an electron pocket at $\boldsymbol{k}=(0,0)$ and a hole pocket at $\boldsymbol{k}=(\pi, \pi)$ of the Brillouin zone. Hence, the modulation vector of the density waves is given by $\boldsymbol{Q}=(\pi, \pi)$. Throughout the paper, we assume the relation $U^{\prime}=(U+J) / 2$ between the interaction parameters so that the Hartree shift can be suppressed. The standard choice $U^{\prime}=U-2 J$ (with $J^{\prime}=J$ ) valid in the atomic limit $37-39$ has also been used to check that the essential features obtained in the present paper do not change (see the Appendix). We moreover assume the value $U / t=8$ at which the excitonic density-wave state is stabilized between the band-insulator and the Mottinsulator states ${ }^{22}$

\section{RESULTS OF CALCULATION}

\section{A. Stability of the excitonic density waves}

First, let us examine the stability of the excitonic CDW and SDW states using the grand potential. In Fig. 11(a), we show the calculated grand potentials of the excitonic CDW and SDW states as a function of the variational parameter $\Delta^{\prime}$. We find that the grand potential has the stationary points at $\Delta^{\prime}=0$ and $\Delta^{\prime} \neq 0$, and the latter is lower in energy, indicating that the excitonic density-wave states are thermodynamically stable. At $J=J^{\prime}=0$, the grand potentials of the excitonic CDW and SDW states are exactly degenerate [see Fig. 11(a)], but Hund's rule coupling $J$ and pair hopping term $J^{\prime}$ lift this degeneracy. The optimized values of the grand potential as a function of $J\left(=J^{\prime}\right)$ are shown in Fig. 1(b) where we find that, with increasing $J$ (and $J^{\prime}$ ), the energy of the excitonic SDW state decreases, but the energy of the excitonic CDW state increases and approaches the energy of the normal semimetallic state. Therefore, the excitonic SDW (CDW) state is stabilized (destabilized) by $J$ and $J^{\prime}$. In Fig. 1(c), we show the optimized values of the grand potentials in the presence $\left(J^{\prime}=J\right)$ and absence $\left(J^{\prime}=0\right)$ of the pair hopping term where we find
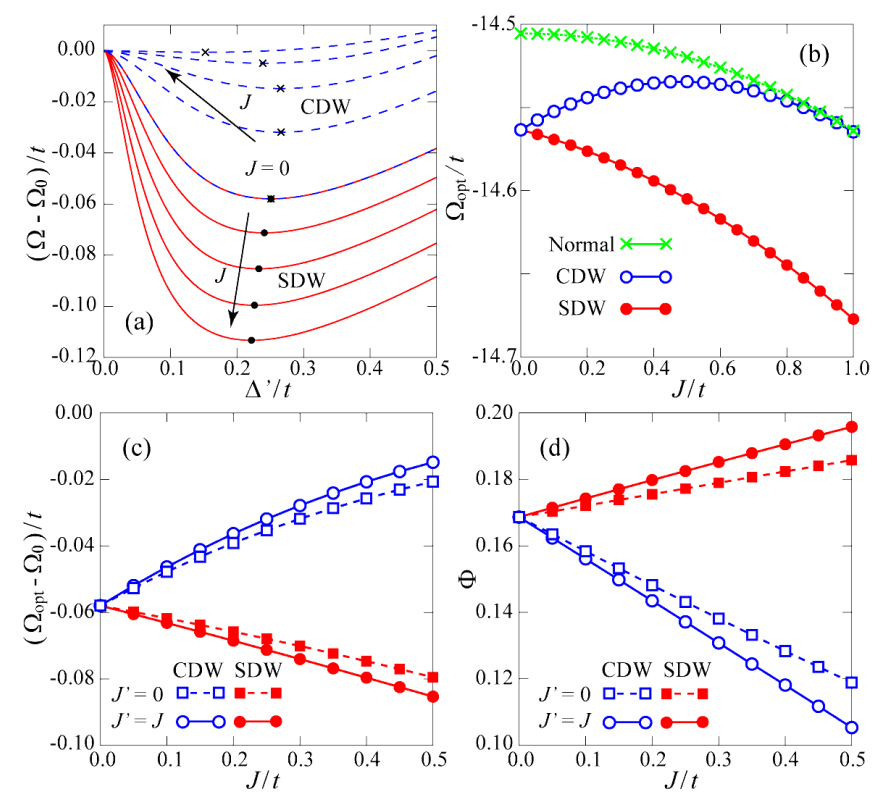

FIG. 1: (Color online) (a) Calculated grand potentials for the excitonic CDW and SDW states as a function of the variational parameter $\Delta^{\prime}\left(=\Delta_{0}^{\prime}, \Delta_{z}^{\prime}\right)$ at $J / t=J^{\prime} / t=0,0.25$, $0.5,0.75$, and 1 . $\Omega_{0}$ is the grand potential in the normal (semimetallic) state. The crosses and dots indicate the stationary points of the excitonic CDW and SDW states, respectively. (b) $J\left(=J^{\prime}\right)$ dependence of the grand potential at the stationary point for the normal (or semimetallic), excitonic CDW, and SDW states. (c) Optimized values of the grand potentials in the presence $\left(J^{\prime}=J\right)$ and absence $\left(J^{\prime}=0\right)$ of the pair hopping term. (d) $J$ dependence of the order parameters of the excitonic CDW and SDW states in the presence $\left(J^{\prime}=J\right)$ and absence $\left(J^{\prime}=0\right)$ of the pair hopping term.

that the stability of the excitonic SDW (CDW) state is enhanced (suppressed) by pair hopping term $J^{\prime}$.

We also calculate the order parameters of the excitonic CDW and SDW states. Here, we introduce the quantities $\Phi_{0}$ and $\Phi_{z}$ for the excitonic CDW and SDW order parameters, respectively, which are defined as

$$
\begin{aligned}
& \Phi_{0}=\frac{1}{2 N} \sum_{\boldsymbol{k}} \sum_{\sigma}\left\langle c_{\boldsymbol{k}+\boldsymbol{Q} \sigma}^{\dagger} f_{\boldsymbol{k} \sigma}\right\rangle, \\
& \Phi_{z}=\frac{1}{2 N} \sum_{\boldsymbol{k}} \sum_{\sigma} \sigma\left\langle c_{\boldsymbol{k}+\boldsymbol{Q} \sigma}^{\dagger} f_{\boldsymbol{k} \sigma}\right\rangle .
\end{aligned}
$$

The calculated results for $\Phi_{0}$ and $\Phi_{z}$ are shown in Fig. 1(d) in the presence $\left(J^{\prime}=J\right)$ and absence $\left(J^{\prime}=0\right)$ of the pair hopping term. We find that $\Phi_{z}$ is enhanced with $J$ (and $J^{\prime}$ ) and $\Phi_{0}$ is suppressed with $J$ (and $J^{\prime}$ ), which are in accordance with the stability of the excitonic CDW and SDW states evaluated from the behaviors of the calculated grand potentials. Thus, we may state that Hund's rule coupling stabilizes the excitonic SDW state and destabilizes the excitonic CDW state. As seen in Figs. 1(c) and 1(d), we may moreover state that the pair hopping term enhances the stability of the excitonic SDW state and suppresses the stability of the excitonic CDW state. 


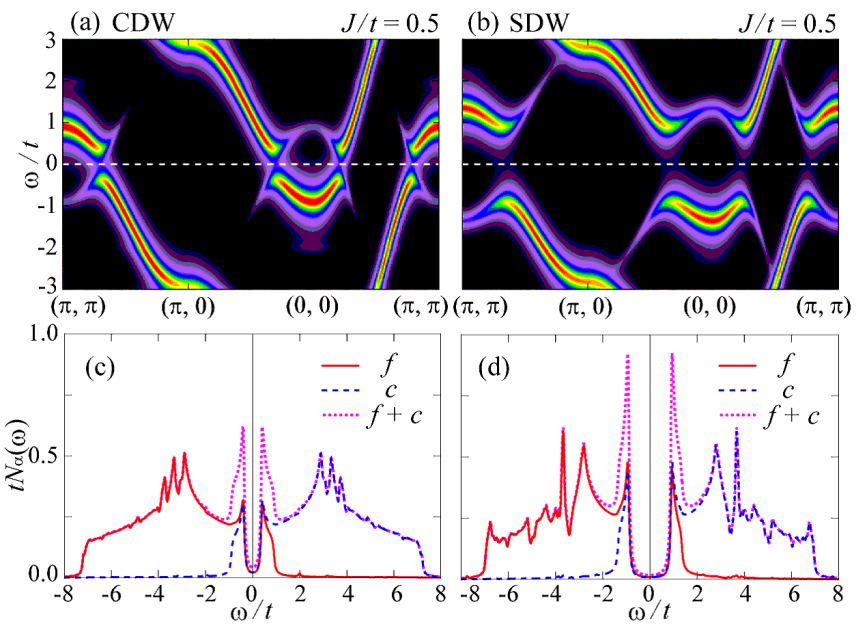

FIG. 2: (Color online) Single-particle spectral function $A(\boldsymbol{k}, \omega)$ and DOS $N_{\alpha}(\omega)$ calculated by CPT at $J / t=J^{\prime} / t=$ 0.5. We show the results for the excitonic CDW state (metastable) in (a) and (c) and for the excitonic SDW state (stable) in (b) and (d). In (c) and (d), the solid, dashed, and dotted lines indicate the $f$ orbital, $c$ orbital, and total DOSs, respectively. The artificial Lorentzian broadening of $\eta / t=0.15$ is used for $A(\boldsymbol{k}, \omega)$ and $\eta / t=0.05$ is used for $N_{\alpha}(\omega)$. The Fermi level is located at $\omega=0$.

\section{B. Single-particle spectral function}

Next, let us calculate the Green's function at the optimized values of the variational parameters using the cluster perturbation theory (CPT) $\stackrel{40}{2}$ The Green's function is defined as

$$
\hat{\mathcal{G}}_{\sigma}\left(\boldsymbol{k}, \boldsymbol{k}^{\prime}, \omega\right)=\frac{1}{L_{c}} \sum_{i, j=1}^{L_{c}} \hat{G}_{i j, \sigma}^{\mathrm{CPT}}(\boldsymbol{k}, \omega) e^{-i \boldsymbol{k} \cdot \boldsymbol{r}_{i}+i \boldsymbol{k}^{\prime} \cdot \boldsymbol{r}_{j}}
$$

where $\hat{G}_{\sigma}^{\mathrm{CPT}}(\boldsymbol{k}, \omega)=\left[\hat{G}_{\sigma}^{\prime-1}(\omega)-\hat{V}_{\sigma}(\boldsymbol{k})\right]^{-1}$. Using this Green's function, the single-particle spectral function is defined as

$$
A(\boldsymbol{k}, \omega)=-\frac{1}{\pi} \sum_{\alpha, \sigma} \operatorname{Im} \mathcal{G}_{\sigma}^{\alpha \alpha}(\boldsymbol{k}, \boldsymbol{k}, \omega+i \eta),
$$

where $\eta$ gives the artificial Lorentzian broadening to the spectrum. We also calculate the DOS for the $\alpha(=f, c)$ orbital, which is defined as

$$
N_{\alpha}(\omega)=-\frac{1}{\pi N} \sum_{\boldsymbol{k}} \sum_{\sigma} \operatorname{Im} \mathcal{G}_{\sigma}^{\alpha \alpha}(\boldsymbol{k}, \omega+i \eta) .
$$

In Fig. 2, we show the calculated single-particle spectral function $A(\boldsymbol{k}, \omega)$ and $\operatorname{DOS} N_{\alpha}(\omega)$; the results for the metastable CDW state [see Figs. 2(a) and 2(c)] and stable SDW state [see Figs. 2(b) and 2(d)] obtained at $J / t=J^{\prime} / t=0.5$ are shown. We find that, although a semimetallic state with a small band overlap is assumed as the noninteracting band structure, the valence band around $\boldsymbol{k}=(\pi, \pi)$ is hybridized with the conduction band around $\boldsymbol{k}=(0,0)$ due to the spontaneous $c$ - $f$ hybridization (or exciton condensation), leading to the opening

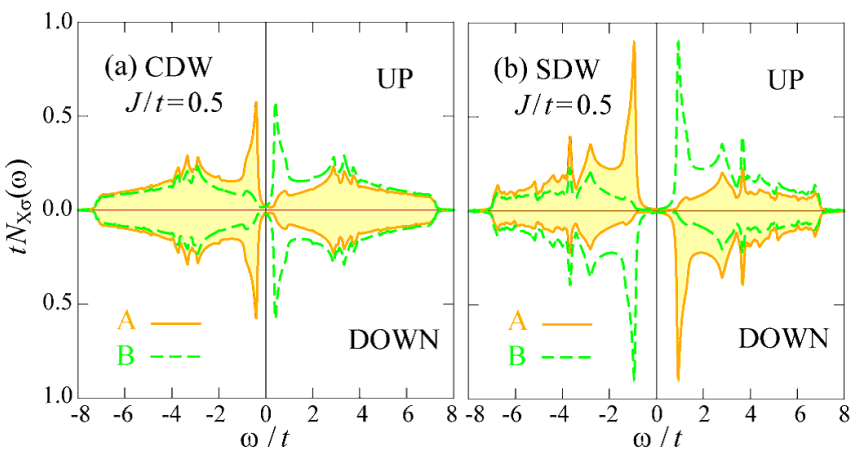

FIG. 3: (Color online) Calculated DOSs for the (a) excitonic CDW state and (b) excitonic SDW state at $J / t=J^{\prime} / t=0.5$. Solid and dashed lines indicate the DOSs of the A and B sublattices, respectively. The Lorentzian broadening of $\eta / t=$ 0.05 is used. The vertical line indicates the Fermi level.

of the band gap at the Fermi level. At $J=J^{\prime}=0$, the single-particle excitation gap $\Delta_{g}$ is estimated to be $\Delta_{g} / t=1.47$. We find that, in agreement with the change in the order parameters, the single-particle gap in the excitonic CDW state, e.g., $\Delta_{g} / t=0.76$ at $J / t=J^{\prime} / t=0.5$, is suppressed in comparison with the $J=J^{\prime}=0$ case [see Figs. 2(a) and 2(c)]. We also find that the single-particle gap in the excitonic SDW state, e.g., $\Delta_{g} / t=1.81$ at $J / t=J^{\prime} / t=0.5$, is enhanced in comparison with the $J=J^{\prime}=0$ case [see Figs. 2(b) and 2(d)]. We moreover find in Figs. 2 (c) and 2(d) that the sharp coherence peak appears at the edges of the gap and that the coherence peak of the SDW state is sharper than that of the CDW state, indicating that the spontaneous $c$ - $f$ hybridization in the excitonic SDW (CDW) state is enhanced (suppressed) by Hund's rule coupling and the pair hopping term. We note that no significant differences are found in the behaviors of $N_{\alpha}(\omega)$ discussed above, even if we switch off the pair hopping term, retaining only Hund's rule coupling.

In order to see the character of the excitonic densitywave states, we calculate the DOS of the A and B sublattices. The sublattice Green's function is given by

$$
\hat{\mathcal{G}}_{X \sigma}(\boldsymbol{k}, \omega)=\frac{2}{L_{c}} \sum_{i, j \in X} \hat{G}_{i j, \sigma}^{\mathrm{CPT}}(\boldsymbol{k}, \omega) e^{-i \boldsymbol{k} \cdot\left(\boldsymbol{r}_{i}-\boldsymbol{r}_{j}\right)}
$$

with $X=\mathrm{A}$ or B. Using this sublattice Green's function, the DOS of the A or B sublattices is defined as

$$
N_{X \sigma}(\omega)=-\frac{1}{\pi N} \sum_{\boldsymbol{k}} \sum_{\alpha, \beta} \operatorname{Im} \mathcal{G}_{X \sigma}^{\alpha \beta}(\boldsymbol{k}, \omega+i \eta) .
$$

In Fig. 3(a), we show the calculated DOS for the excitonic CDW state at $J / t=J^{\prime} / t=0.5$. We note that, below the Fermi level $(\omega<0)$, the up- and down-spin DOSs are the same and the DOS of the A sublattice is larger than that of the B sublattice: $N_{A \uparrow}(\omega)=N_{A \downarrow}(\omega)>$ $N_{B \uparrow}(\omega)=N_{B \downarrow}(\omega)$. We also note that $N_{A \sigma}(\omega) \simeq N_{B \sigma}(\omega)$ far away from the Fermi level and that the coherence peak appears in the DOS of the A sublattice just below the Fermi level, where $N_{A \sigma}(\omega)>N_{B \sigma}(\omega)$. Using the order parameter $\Phi_{0}$, the local number of electrons is given by $n_{i}=1+2 \Phi_{0} \cos \left(\boldsymbol{Q} \cdot \boldsymbol{r}_{i}\right)$. At $J / t=J^{\prime} / t=0.5$, we have 

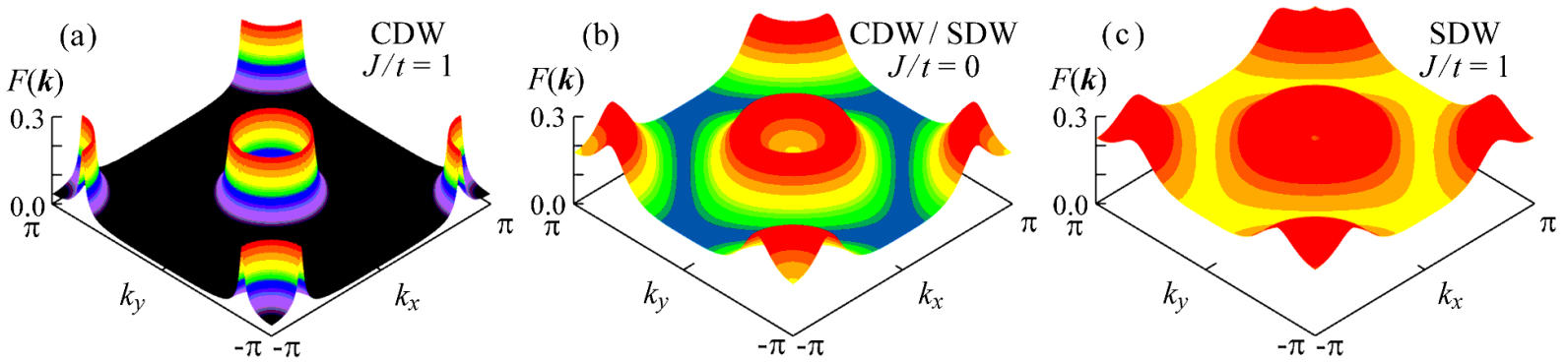

FIG. 4: (Color online) Condensation amplitude $F(\boldsymbol{k})\left[=F_{0}(\boldsymbol{k})\right.$ or $\left.F_{z}(\boldsymbol{k})\right]$ calculated by CPT. We show the results for (a) the CDW state at $J / t=J^{\prime} / t=1.0$ (metastable), (b) the CDW/SDW states at $J=J^{\prime}=0$ (degenerate), and (c) the SDW state at $J / t=J^{\prime} / t=1.0$ (stable).

$\Phi_{0}=0.11$, and thus the local numbers of the electrons on each sublattice are given by $n_{A \uparrow}=n_{A \downarrow}=1+2 \Phi_{0}=1.22$ and $n_{B \uparrow}=n_{B \downarrow}=1-2 \Phi_{0}=0.78$. We therefore find that, due to the effect of Hund's rule coupling and the pair hopping term, $\Phi_{0}$ is suppressed and thus the excitonic CDW modulation in real space becomes rather weak.

In Fig. 3(b), we show the calculated DOS for the excitonic SDW state at $J / t=J^{\prime} / t=0.5$. We note that, below Fermi level, the up-spin DOS of the A (B) sublattice is equal to the down-spin DOS of the B (A) sublattice and that the up-spin DOS of the A (B) sublattice is larger (smaller) than the down-spin DOS of the A (B) sublattice: $N_{A \uparrow}(\omega)=N_{B \downarrow}(\omega)>N_{A \downarrow}(\omega)=N_{B \uparrow}(\omega)$. We also note that the DOS has a large gap and a sharp coherence peak appears at the edge of the DOS. Using the order parameter $\Phi_{z}$, the local magnetization is given by $m_{i}=2 \Phi_{z} \cos \left(\boldsymbol{Q} \cdot \boldsymbol{r}_{i}\right)$. At $J / t=J^{\prime} / t=0.5$, we have $\Phi_{z}=0.20$, and thus the local numbers of electrons on each sublattice are given by $n_{A \uparrow}=n_{B \downarrow}=1+2 \Phi_{z}=1.40$ and $n_{A \downarrow}=n_{B \uparrow}=1-2 \Phi_{z}=0.60$. We therefore find that, due to the effect of Hund's rule coupling and the pair hopping term, $\Phi_{z}$ is enhanced, and thus the excitonic SDW modulation in real space becomes rather strong.

\section{Condensation amplitude and coherence length}

In order to see the character of the exciton condensation in momentum space, we calculate the condensation amplitude (or the anomalous momentum distribution function). Using the off-diagonal (or anomalous) Green's function given in Eq. (14), the condensation amplitudes for the spin-singlet and spin-triplet excitons are defined as

$$
\begin{aligned}
& F_{0}(\boldsymbol{k})=\frac{1}{2} \sum_{\sigma} \oint_{C} \frac{\mathrm{d} z}{2 \pi i} \mathcal{G}_{\sigma}^{c f}(\boldsymbol{k}, \boldsymbol{k}+\boldsymbol{Q}, z), \\
& F_{z}(\boldsymbol{k})=\frac{1}{2} \sum_{\sigma} \sigma \oint_{C} \frac{\mathrm{d} z}{2 \pi i} \mathcal{G}_{\sigma}^{c f}(\boldsymbol{k}, \boldsymbol{k}+\boldsymbol{Q}, z),
\end{aligned}
$$

respectively. Note that we here use the term "anomalous" to indicate that the number of electrons on each of the $c$ and $f$ orbitals is not conserved due to the excitonic condensation, although the total number of electrons is conserved.
We show the calculated results in Fig. 4 for the excitonic CDW and SDW states. We find that, with increasing $J\left(=J^{\prime}\right)$, the peak of $F_{0}(\boldsymbol{k})$ at the Fermi momentum $\boldsymbol{k}_{\mathrm{F}}$ becomes sharper in the CDW state [see Fig. [4(a)] and that the peak of $F_{z}(\boldsymbol{k})$ at $\boldsymbol{k}_{\mathrm{F}}$ becomes broader in momentum space in the SDW state [see Fig. 4(c)]. The sharp (broad) peak of $F(\boldsymbol{k})\left[=F_{0}(\boldsymbol{k})\right.$ or $\left.F_{z}(\boldsymbol{k})\right]$ in momentum space indicates that the spatial extension of the electronhole pair becomes large (small) in real space. We note that no significant differences are found in the behavior of $F(\boldsymbol{k})$, even if we set $J^{\prime}=0$ retaining only Hund's rule coupling.

Using $F(\boldsymbol{k})$, we evaluate the pair coherence length $\xi$, which corresponds to the spatial size of the electron-hole pair and may be defined by $26,28,29$

$$
\xi^{2}=\frac{\sum_{\boldsymbol{k}}\left|\nabla_{\boldsymbol{k}} F(\boldsymbol{k})\right|^{2}}{\sum_{\boldsymbol{k}}|F(\boldsymbol{k})|^{2}} .
$$

In Fig. 5 we show the calculated results for the spinsinglet excitons $\left(\xi_{0}\right)$ and spin-triplet excitons $\left(\xi_{z}\right)$ as a function of $J$. We find that, with increasing $J\left(=J^{\prime}\right), \xi$ for the spin-singlet (triplet) excitons increases (decreases) monotonically. Thus, the size of the spin-singlet exciton becomes larger than the lattice constant $\left(\xi_{0}>1\right)$ for larger $J$ values, indicating the crossover from the tightly paired BEC state to the weakly paired BCS state. The spin-triplet excitons, on the other hand, are paired more tightly, and the size is always smaller than the lattice constant in the parameter space examined. We also find in the inset of Fig. 5 that the above tendencies induced by Hund's rule coupling are again enhanced by the pair hopping term.

\section{SUMMARY AND DISCUSSION}

To summarize, we have studied the stability of the excitonic density-wave states in the two-band Hubbard model with the interorbital Coulomb interaction $U^{\prime}$, Hund's rule coupling $J$, pair hopping term $J^{\prime}$, as well as the intraorbital Hubbard interaction $U$. We have rewritten the interorbital interactions of the Hamiltonian in terms of the creation and annihilation operators of the spin-singlet and spin-triplet excitons and examined the roles of these interactions. We have thereby shown that the $U^{\prime}$ term drives the formation of excitons in both the spin-singlet and the spin-triplet channels, and the 


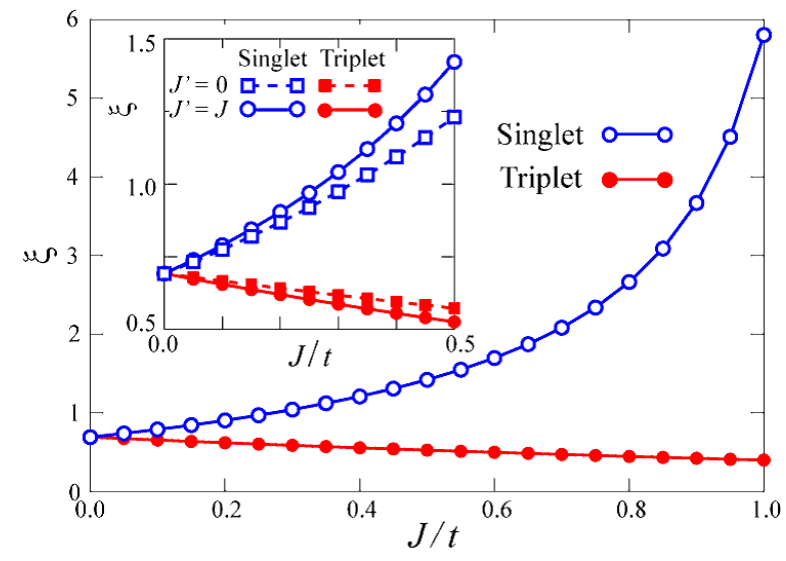

FIG. 5: (Color online) Calculated pair coherence length $\xi$ in units of the lattice constant. $J\left(=J^{\prime}\right)$ dependence of $\xi$ is shown for the spin-singlet (open circles) and spin-triplet (solid circles) exciton condensations. The inset shows the results in the absence of the pair hopping term $J^{\prime}=0$ (open and solid squares), which are compared with the results in the presence of the pair hopping term $J^{\prime}=J$ (open and solid circles).

$J$ term stabilizes (destabilizes) the formation of the spintriplet (spin-singlet) excitons. Using the VCA to calculate the grand potential of the system in the thermodynamic limit, we have moreover shown that Hund's rule coupling always stabilizes the excitonic SDW state and destabilizes the excitonic CDW state of which the tendencies are enhanced by the pair hopping term. A variety of physical quantities has also been calculated, which include the single-particle spectral function, density of states, anomalous Green's functions, condensation amplitude, and pair coherence length. We have thus characterized the excitonic CDW and SDW states in detail.

Finally, let us discuss the experimental implications of our results obtained in this paper. At first sight, the condensations of the spin-singlet excitons possibly observed in $1 T$-TiSe 2 (Refs. [13 16]) and $\mathrm{Ta}_{2} \mathrm{NiSe}_{5}$ (Refs. [9 12]) seem to contradict the stability of the spin-triplet excitons in the presence of Hund's rule coupling. However, in these materials, the valence and conduction bands are formed by the orbitals located on different atoms, i.e., the $4 p$ orbitals of Se ions for the valence bands and the $3 d$ orbitals of $\mathrm{Ti}$ ions for the conduction bands in $1 T$ $\mathrm{TiSe}_{2}, \frac{13}{3}$ and the $3 d$ orbitals of $\mathrm{Ni}$ ions for the valence bands, and the $5 d$ orbitals of Ta ions for the conduction bands in $\mathrm{Ta}_{2} \mathrm{NiSe}_{5}, \frac{11}{=}$ and therefore Hund's rule coupling acting between electrons on different orbitals in a single ion does not work to stabilize the condensation of the spin-triplet excitons. We anticipate that in these materials the electron-phonon coupling should work to stabilize the condensation of the spin-singlet excitons as was discussed in Refs. [11,15, 16]. In the excitonic SDW states possibly observed in, e.g., iron pnictide superconductors and Co oxide materials, on the other hand, Hund's rule coupling rather than the electron-phonon coupling should work to stabilize the condensation of the spin-triplet excitons as we have shown in this paper. We may therefore suggest that the competition between Hund's rule coupling and electron-phonon coupling in the stability of excitonic condensations (or excitonic density-wave for-
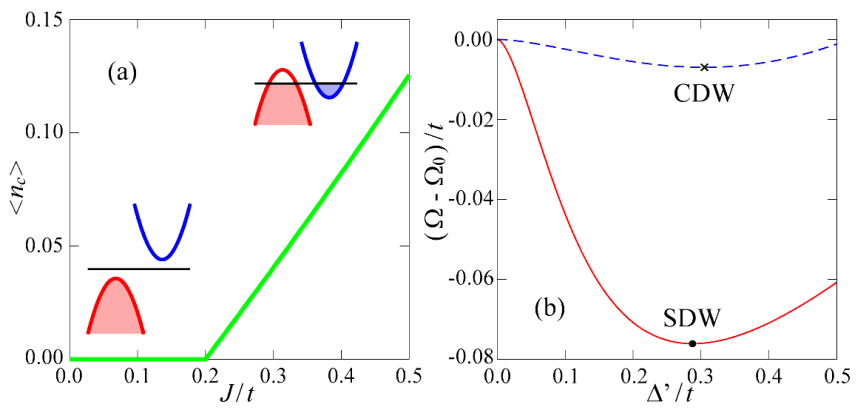

FIG. 6: (Color online) (a) The number of the conduction-band electrons $\left\langle n_{c}\right\rangle$ (or the valence-band holes) as a function of $\mathrm{J} / \mathrm{t}$ in the normal state (or $\Delta^{\prime}=0$ ), which is obtained using the atomic-limit relation $U^{\prime}=U-2 J$ with $U / t=5, D / t=2$, and $J^{\prime}=0$. (b) Calculated grand potentials of the excitonic CDW and SDW states as a function of the variational parameter $\Delta^{\prime}$ $\left(=\Delta_{0}^{\prime}, \Delta_{z}^{\prime}\right)$, which are obtained using the atomic-limit relation $U^{\prime}=U-2 J$ with $U / t=5, J / t=J^{\prime} / t=0.5$ and $D / t=2$. The crosses and circles indicate the stationary points of the excitonic CDW and SDW states, respectively.

mations) will be of great interest in future studies.

\section{Acknowledgments}

T. K. acknowledges support from the JSPS Research Fellowship for Young Scientists. This work was supported, in part, by a Kakenhi Grant No. 26400349 from JSPS of Japan.

\section{Appendix A: Use of the atomic-limit relation}

In the main text, we have assumed the relation $U^{\prime}=$ $(U+J) / 2$ between the interaction parameters. In this Appendix, we present some results obtained in a different choice of the relation, i.e., $U^{\prime}=U-2 J$, which is valid in the atomic limit, $\stackrel{37-39}{-39}$ and show that the essential features of our results do not alter.

In the BCS-like mean-field theory applied to our model Eq. (11), the diagonal terms of the mean-field Hamiltonian are given by $\varepsilon_{f}(\boldsymbol{k})=-\varepsilon_{c}(\boldsymbol{k})=-2 t \sum_{i}^{d} \cos k_{i}-D+$ $n\left(U / 2-U^{\prime}+J / 2\right)$ with $n=\left\langle n_{i f \sigma}\right\rangle-\left\langle n_{i c \sigma}\right\rangle$, and the off-diagonal term gives the spontaneous $c-f$ hybridization (or excitonic condensation) 11 The Hartree shift $n\left(U / 2-U^{\prime}+J / 2\right)$ appears in this expression. Depending on the values of $U, U^{\prime}$ and $J$, we therefore find, e.g., the Mott-insulator state at $U^{\prime} \ll(U+J) / 2$ and the bandinsulator state at $U^{\prime} \gg(U+J) / 2, \frac{19,22}{2}$ which are due simply to the effect of the Hartree shift.

The effects of this Hartree shift can be suppressed completely if we assume the relation $U^{\prime}=(U+J) / 2$ as in the main text. However, if we assume the atomic-limit relation $U^{\prime}=U-2 J$, the change in the parameter values, e.g., $J$, leads to the change in the overlap of the valence and conduction bands and hence to the change in the number of conduction-band electrons (and valence-band holes) as shown in Fig. 6(a). This gives an additional complexity to our calculations because in this paper we just want to focus on the relative stability of the exci- 
tonic CDW and SDW states in the presence of Hund's rule coupling and the pair hopping term.

Our assumption of the use of the relation $U^{\prime}=(U+$ $J) / 2$ may be justified if the essential features obtained in the main text do not differ from the results obtained using the atomic-limit relation $U^{\prime}=U-2 J$. In Fig. 6(b), we show the grand potentials as a function of the vari- ational parameter $\Delta^{\prime}$ calculated using the atomic-limit relation $U^{\prime}=U-2 J$ where we actually find that the results are nearly the same as the results shown in Fig. 1(a) in the main text. Therefore, we may safely state that the essential features obtained in the main text do not alter in the different choice of the parameter set.
1 D. Jérome, T. M. Rice, and W. Kohn, Phys. Rev. 158, 462 (1967).

2 B. I. Halperin and T. M. Rice, Rev. Mod. Phys. 40, 755 (1968).

3 B. I. Halperin and T. M. Rice, in Solid State Physics, edited by F. Seitz, D. Turnbull, and H. Ehrenreich (Academic, New York, 1968), Vol. 21, p. 115.

${ }^{4}$ F. X. Bronold and H. Fehske, Phys. Rev. B 74, 165107 (2006).

5 B. Bucher, P. Steiner, and P. Wachter, Phys. Rev. Lett. 67, 2717 (1991).

6 D. P. Young, D. Hall, M. E. Torelli, Z. Fisk, J. L. Sarrao, J. D. Thompson, H.-R. Ott, S. B. Oseroff, R. G. Goodrich, and R. Zysler, Nature (London) 397, 412 (1999).

7 M. E. Zhitomirsky, T. M. Rice, and V. I. Anisimov, Nature (London) 402, 251 (1999).

8 L. Balents and C. M. Varma, Phys. Rev. Lett. 84, 1264 (2000).

9 Y. Wakisaka, T. Sudayama, K. Takubo, T. Mizokawa, M. Arita, H. Namatame, M. Taniguchi, N. Katayama, M. Nohara, and H. Takagi, Phys. Rev. Lett. 103, 026402 (2009).

10 Y. Wakisaka, T. Sudayama, K. Takubo, T. Mizokawa, N. L. Saini, M. Arita, H. Namatame, M. Taniguchi, N. Katayama, M. Nohara, and H. Takagi, J. Supercond. Novel Magn. 25, 1231 (2012).

11 T. Kaneko, T. Toriyama, T. Konishi, and Y. Ohta, Phys. Rev. B. 87, 035121 (2013); 87, 199902(E) (2013).

12 K. Seki, Y. Wakisaka, T. Kaneko, T. Toriyama, T. Konishi, T. Sudayama, N. L. Saini, M. Arita, H. Namatame, M. Taniguchi, N. Katayama, M. Nohara, H. Takagi, T. Mizokawa, and Y. Ohta, Phys. Rev. B 90, 155116 (2014).

13 H. Cercellier, C. Monney, F. Clerc, C. Battaglia, L. Despont, M. G. Garnier, H. Beck, P. Aebi, L. Patthey, H. Berger, and L. Forró, Phys. Rev. Lett. 99, 146403 (2007).

14 C. Monney, H. Cercellier, F. Clerc, C. Battaglia, E. F. Schwier, C. Didiot, M. G. Garnier, H. Beck, P. Aebi, H. Berger, L. Forró, and L. Patthey, Phys. Rev. B. 79, 045116 (2009).

15 C. Monney, C. Battaglia, H. Cercellier, P. Aebi, and H. Beck, Phys. Rev. Lett. 106, 106404 (2011).

16 B. Zenker, H. Fehske, H. Beck, C. Monney, and A. R. Bishop, Phys. Rev. B. 88, 075138 (2013).

17 P. M. R. Brydon and C. Timm, Phys. Rev. B 79, 180504(R) (2009).

18 P. M. R. Brydon and C. Timm, Phys. Rev. B 80, 174401 (2009).
19 B. Zocher, C. Timm, and P. M. R. Brydon, Phys. Rev. B 84, 144425 (2011).

20 J. Kuneš and P. Augustinský, Phys. Rev. B 89, 115134 (2014)

21 J. Kuneš and P. Augustinský, Phys. Rev. B 90, 235112 (2014).

22 T. Kaneko, K. Seki, and Y. Ohta, Phys, Rev. B 85, 165135 (2012).

23 M. Potthoff, M. Aichhorn, and C. Dahnken, Phys. Rev. Lett 91, 206402 (2003).

24 C. Dahnken, M. Aichhorn, W. Hanke, E. Arrigoni, and M. Potthoff, Phys. Rev. B 70, 245110 (2004).

25 C. D. Batista, Phys. Rev. Lett. 89, 166403 (2002).

${ }^{26}$ K. Seki, R. Eder, and Y. Ohta, Phys. Rev. B 84, 245106 (2011).

27 B. Zenker, D. Ihle, F. X. Bronold, and H. Fehske, Phys. Rev. B 85, 121102(R) (2012).

28 T. Kaneko, S. Ejima, H. Fehske, and Y. Ohta, Phys, Rev. B 88, 035312 (2013).

29 S. Ejima, T. Kaneko, Y. Ohta, and H. Fehske, Phys. Rev. Lett 112, 026401 (2014).

${ }^{30}$ For a recent review, see, e.g., E. Dagotto, A. Moreo, A. Nicholson, Q. Luo, S. Liang, and X. Zhang, Front. Phys. 6, 379 (2011);

31 P. M. R. Brydon, M. Daghofer, and C. Timm, J. Phys.: Condens. Matter 23, 246001 (2011), and references therein.

32 M. Daghofer, A. Nicholson, A. Moreo, and E. Dagotto, Phys. Rev. B 81, 014511 (2010).

33 Q. Luo, G. Martins, D.-X. Yao, M. Daghofer, R. Yu, A. Moreo, and E. Dagotto, Phys. Rev. B 82, 104508 (2010).

34 M. Potthoff, Eur. Phys. J. B 32, 429 (2003); 36, 335 (2003).

35 M. Potthoff, in Strongly Correlated Systems - Theoretical Methods, edited by A. Avella and F. Mancini, Springer Series in Solid-State Sciences Vol. 171 (Springer, Berlin, 2012), pp. 303-339.

36 D. Sénéchal, e-print arXiv:0806.2690

37 J. S. Griffith, The Theory of Transition-Metal Ions (Cambridge University Press, Cambridge, U.K., 1961).

38 B. H. Brandow, Adv. Phys. 26, 651 (1977).

39 A. M. Olés, Phys. Rev. B 28, 327 (1983).

40 D. Sénéchal, D. Perez, and M. Pioro-Ladriere, Phys. Rev. Lett 84, 522 (2000). 